\title{
DIFFUSION IN TURBULENT FLOW BETWEEN PARALLEL PLANES*
}

\author{
BY \\ J. C. JAEGER \\ University of Tasmania
}

1. Introduction. The equation of diffusion in a turbulent fluid

$$
\frac{\partial^{2} \chi}{\partial z^{2}}+\frac{1-2 p}{z} \frac{\partial \chi}{\partial z}=\frac{\partial \chi}{\partial x}
$$

( $\chi$ stands for temperature, vapour concentration, or whatever property is being studied, $x$ is measured in the direction of mean flow and $z$ in the perpendicular direction, and $p$ is a constant determined by the degree of turbulence of the fluid) was introduced by O. G. Sutton ${ }^{1}$ and extensively studied by W. G. L. Sutton, ${ }^{2}$ who considered a number of cases of diffusion in the semi-infinite region $z>0$. It has been shown by Pasquill ${ }^{3}$ that for the semi-infinite region the theory is in good agreement with experiments, both on evaporation and on heat transfer.

In this note a number of results for symmetrical flow in the finite region $0<z<2 l$ will be given; it is assumed that $2 l$ is small enough for the power law velocity profile to hold up to the centre of the region. Such cases are of some practical interest, and may provide an indication of the behaviour to be expected in the much more difficult problem of heat transfer in a circular pipe. Also they are interesting generalizations of known solutions of the equation of conduction of heat in the rod $0<z<2 l$, with constant temperature, or flow of heat, at its ends.

The method used will be that of the Laplace transformation. W. G. L. Sutton (loc. cit.) remarks that if $p=1 / 2$, equation (1) reduces to the equation of linear flow of heat, and he gives a treatment of (1) which is a generalization of Goursat's treatment of the equation of conduction of heat. It is well known that the Laplace transformation method is particularly well suited to the solution of specific problems in conduction of heat, and that its advantage increases as the complexity of the problem increases. This suggests that the method may have the same advantages when applied to (1), and, in fact, this proves to be the case. All the results of W. G. L. Sutton's paper can be obtained more shortly in this way, and explicit expressions for the solutions for more complicated boundary conditions, composite regions, etc., can also be derived.

In Section 2 the standard problem of evaporation in the semi-infinite region is solved as an illustration of the method, and for comparison with later results. In

* Received Dec. 26, 1944.

1 O. G. Sutton, Wind structure and evaporation in a turbulent atmosphere, Proc. Roy. Soc. (A), 146, 701 (1934).

2 W. G. L. Sutton, On the equation of diffusion in a turbulent medium, Proc. Roy. Soc. (A), 182, 48 (1943). The notation used here is that of this paper, except that the symbol $E$ is introduced in (4). The variables $x$ and $z$ in (1) are dimensionless quantities defined in Sutton's paper.

' F. Pasquill, Evaporation from a plane, free-liquid surface into a turbulent air stream, Proc. Roy. Soc. (A), 182, 75 (1943). 
Section 3 two other results for the semi-infinite region are given for completeness. In Sections 4-6 the most interesting cases of symmetrical flow in the region $0<z<2 l$ are studied. The solutions given here are formal only, but in all cases they may be made rigorous by the verification process described elsewhere. ${ }^{4}$

Equation (1) has to be solved in the region $x>0$, and in a prescribed region of $z$, with boundary conditions in $x$

$$
\begin{aligned}
& \chi \rightarrow \chi^{(0)}(z), \text { as } x \rightarrow+0, \\
& \chi \text { finite, } \quad \text { as } x \rightarrow \infty .
\end{aligned}
$$

In all the problems considered below $\chi^{(0)}(z)$ will be zero, that is the temperature or vapour concentration in the fluid is zero in the plane $x=0$.

There are also boundary conditions in $z$, which will be expressed either in terms of $\chi$, or of

$$
E=-B z^{1-2 p} \frac{\partial \chi}{\partial z}
$$

This quantity $E$ is the local rate of diffusion across the plane $z=$ const., and $B$ is a known constant (defined by Sutton, loc. cit.) involving the fluid and its degree of turbulence.

The constant $p$ in (1) is restricted in Sutton's theory by the inequality $0<p<1 / 3$, and we assume here $0<p<1$.

With the substitution

$$
\chi=z^{p} \Omega,
$$

(1) becomes

$$
\frac{\partial^{2} \Omega}{\partial z^{2}}+\frac{1}{z} \frac{\partial \Omega}{\partial z}-\frac{p^{2}}{z^{2}} \Omega-\frac{\partial \Omega}{\partial x}=0 .
$$

Introducing the Laplace transform of $\Omega$ with respect to $x$, namely

$$
\Omega^{*}=\int_{0}^{\infty} e^{-s x} \Omega d x
$$

we obtain from (6) the subsidiary equation ${ }^{5}$ for $\Omega^{*}$,

$$
\frac{d^{2} \Omega^{*}}{d z^{2}}+\frac{1}{z} \frac{d \Omega^{*}}{d z}-\left(s+\frac{p^{2}}{z^{2}}\right) \Omega^{*}=-z^{-p} \chi^{(0)}(z) .
$$

2. The semi-infinite region $z>0$. Boundary conditions: $\chi^{(0)}(z)=0, z>0 . \chi=\chi_{0}$, constant, ${ }^{6}$ when $z=0, x>0$. $\chi$ finite, as $z \rightarrow \infty, x>0$. Here (8) becomes

$$
\frac{d^{2} \Omega^{*}}{d z^{2}}+\frac{1}{z} \frac{d \Omega^{*}}{d z}-\left(s+\frac{p^{2}}{z^{2}}\right) \Omega^{*}=0, \quad z>0,
$$

${ }^{4} \mathrm{H}$. S. Carslaw and J. C. Jaeger, Operational methods in applied mathematics, Oxford, 1941, \$58, and J. C. Jaeger, Radial heat flow in a circular cylinder with a general boundary condition, Proc. Roy. Soc. N.S.W., 75, 130-139 (1942).

G For the procedure see, e.g., Carslaw and Jaeger, loc. cit.

- For shortness, boundary conditions will usually be written in this way; it is implied, of course, that $x \rightarrow \chi_{0}$ as $z \rightarrow+0$ for fixed $x>0$. 
to be solved with

$$
\chi^{*}=z^{p} \Omega^{*} \rightarrow \chi_{0} / s, \quad \text { as } \quad z \rightarrow+0,
$$

and

$$
\chi^{*} \text { finite as } z \rightarrow \infty .
$$

The solution of (9) which satisfies (11) is $K_{p}\left(z s^{\frac{1}{2}}\right)$, and since

$$
z^{p} K_{p}\left(z s^{\frac{1}{2}}\right) \rightarrow 2^{p-1} \Gamma(p) s^{-\frac{1}{p} p} \quad \text { as } \quad z \rightarrow+0,
$$

it follows that the solution of (9), (10), and (11) is

$$
\chi^{*}=\chi_{0} \frac{z^{p} 2^{1-p}}{\Gamma(p)} s^{\frac{1}{p-1}} K_{p}\left(z s^{\frac{1}{2}}\right) .
$$

Now it is known that ${ }^{7}$

$$
s^{\frac{1}{p-1}} K_{p}\left(z s^{\frac{1}{3}}\right) \text { is the Laplace transform of } z^{-p} 2^{p-1} \int_{z^{2} / 4 x}^{\infty} e^{-u} u^{p-1} d u \text {. }
$$

Thus the required solution is

$$
\chi=\chi_{0} \frac{1}{\Gamma(p)} \int_{z^{2} / 4 x}^{\infty} e^{-u} u^{p-1} d u .
$$

This is the result given by Pasquill (loc. cit., (9)).

It follows from (15), or directly from the transform $E^{*}$ of $E$, that

$$
E \rightarrow \frac{B \chi_{0} 2^{1-2 p} x^{-p}}{\Gamma(p)}, \quad \text { as } z \rightarrow+0,
$$

and

$$
\int_{0}^{x} E d x \rightarrow \frac{B \chi_{0} 2^{1-2 p} x^{1-p}}{(1-p) \Gamma(p)}, \quad \text { as } \quad z \rightarrow+0 .
$$

3. Two other results for the semi-infinite region $z>0$. The results to be derived here are both for the case $\chi^{(0)}(z)=0$, and $\chi(z)$ finite as $z \rightarrow \infty$.

If the boundary condition at $z=0$ is: $E \rightarrow E_{0}$, constant, as $z \rightarrow+0$, the solution is

Also

$$
\chi=\frac{E_{0} z^{2 p}}{2 B \Gamma(1-p)} \int_{z^{2} / 4 x}^{\infty} e^{-u} u^{-p-1} d u
$$

$$
\chi \rightarrow \frac{E_{0} 2^{2 p-1} x^{p}}{B p \Gamma(1-p)}, \quad \text { as } \quad z \rightarrow+0 .
$$

This is proved exactly as in Section 2, using (14).

${ }^{7} \mathrm{~S}$. Goldstein, Operational representations of Whittaker's confluent hypergeometric function and Weber's parabolic cylinder function, Proc. London Math. Soc. (2) 34, 104 (1932), (15) and (24). Alternatively the result can be obtained by the use of the inversion theorem for the Laplace transformation, subsequently deforming the line integral into the contour $(-\infty, 0+)$; cf. Carslaw and Jaeger, loc. cit., \$39. The same remark applies to the derivation of (18) and (21) below. 
If the boundary condition at $z=0$ is

$$
h \chi-z^{1-2 p} \frac{\partial \chi}{\partial z}=h \chi_{0},
$$

where $h$ and $\chi_{0}$ are constants, the solution is

$$
\chi=\chi_{0}-\frac{\chi_{0} z^{p}}{2^{p-1} \Gamma(p)} \int_{0}^{\infty} e^{-x u^{2} u^{p-1}} \frac{J_{p}(u z)+\alpha u^{2 p} J_{-p}(u z)}{1+2 \alpha u^{2 p} \cos p \pi+\alpha^{2} u^{4 p}} d u,
$$

where $\alpha=\Gamma(1-p) 2^{1-2 p} / h \Gamma(p)$.

To prove (21) the inversion theorem for the Laplace transformation, (24) below, has to be used, and the line integral must be deformed into $(-\infty, 0+)$.

The result (15) was derived for a constant value of $\chi$ on the boundary $z=0$. The solution for the case in which $\chi$ is a prescribed function of $x$ on $z=0$ can be obtained from (15) by Duhamel's theorem. The same remark applies to the cases of Sections 4 and 6. Correspondingly, the solutions of the problems of Sections 3, 5 with $E$ a prescribed function of $x$ on $z=0$ can be obtained in the same way.

4. The region $0<z<l . \chi^{(0)}(z)=0 . \chi=\chi_{0}$, constant, when $z=0, x>0 . E=0$, when $z=l, x>0$. This corresponds to the region $0<z<2 l$ with flow symmetrical about $z=l$, and with $\chi=\chi_{0}$ on $z=0$ and $z=2 l$, for $x>0$. Thus, for example, it gives the solution of the problem of heat transfer from the parallel planes $z=0$ and $z=2 l$, both maintained at constant temperature $\chi_{0}$, and with symmetrical flow between them.

Here we have to solve (9) with boundary conditions (10) and

$$
E^{*}=0 \text {, when } z=l \text {. }
$$

By (12) the solution of (9) which satisfies (10) is

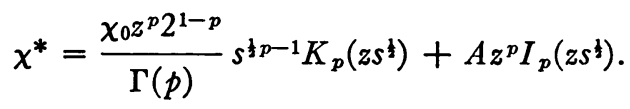

The unknown $A$ is found by substituting in (22), and we have finally

$$
\chi^{*}=\frac{\chi_{\theta} z^{p^{1-p_{s}}} s^{\frac{1}{1}-1}\left[K_{p}\left(z s^{\frac{1}{1}}\right) I_{p-1}\left(l s^{\frac{1}{1}}\right)+I_{p}\left(z s^{\frac{1}{1}}\right) K_{p-1}\left(l s^{\frac{1}{2}}\right)\right]}{\Gamma(p) I_{p-1}\left(l s^{\frac{1}{1}}\right)} .
$$

$\chi$ is found from (23) by using the inversion theorem for the Laplace transformation [cf. Carslaw and Jaeger, loc. cit.]

$$
\begin{aligned}
\chi & =\frac{1}{2 \pi i} \int_{\gamma-i \infty}^{\gamma+i \infty} e^{s x} \chi^{*}(s) d s \\
& =\frac{\chi_{0} z^{p} 2^{1-p}}{2 \pi i \Gamma(p)} \int_{\gamma-i \infty}^{\gamma+i \infty} \frac{e^{s x} s^{\frac{3}{p-1}}\left[K_{p}\left(z s^{\sharp}\right) I_{p-1}\left(l s^{\sharp}\right)+I_{p}\left(z s^{\sharp}\right) K_{p-1}\left(l s^{\sharp}\right)\right] d s}{I_{p-1}\left(l s^{3}\right)},
\end{aligned}
$$

where $\gamma>0$.

The integrand of (25) is a single valued function ${ }^{8}$ of $s$. It has a simple pole at $s=0$ of residue

$$
2^{p-1} z^{-p} \Gamma(p),
$$

${ }^{8} \mathrm{G}$. N. Watson, Theory of Bessel functions, Cambridge University Press, Cambridge 1922, \$3.71, (17) and (18) 
and simple poles at $s=-\alpha_{r}^{2} / l^{2}$, where $\pm \alpha_{r}, r=1,2, \cdots$, are the zeros (all real and simple ${ }^{9}$ ) of

$$
J_{p-1}(\alpha)=0 .
$$

It is easy to show [cf. Jaeger, loc. cit.] that the line integral in (25) is equal to $2 \pi i$ times the sum of the residues at the poles of its integrand. Evaluating these we get finally

$$
\chi=\chi_{0}-\frac{\chi_{0} z^{p}}{2^{p-2} l^{p} \Gamma(p)} \sum_{r=1}^{\infty} \frac{\alpha_{r}^{p-2} e^{-\alpha_{r} x / l^{2}} J_{p}\left(z \alpha_{r} / l\right)}{J_{p}^{2}\left(\alpha_{r}\right)} .
$$

The most interesting quantity is the value of $E$ as $z \rightarrow 0$. Either from (28), or directly by calculation of its transform, this is found to be

$$
E \rightarrow \frac{B \chi_{0} \Gamma(1-p)}{\Gamma(p) 2^{2 p-2} l^{2 p}} \sum_{r=1}^{\infty} \frac{e^{-\alpha_{r}^{2} x / l^{2} J_{1-p}\left(\alpha_{r}\right)}}{\alpha_{r}^{1-2 p} J_{p}\left(\alpha_{r}\right)}, \quad \text { as } \quad z \rightarrow+0 .
$$

Also, as $z \rightarrow+0$,

where

$$
\int_{0}^{x} E d x \rightarrow \frac{B \chi_{0} l^{2-2 p}}{2(1-p)} \phi_{p}^{(1)}\left(x / l^{2}\right)
$$

$$
\phi_{p}^{(1)}\left(x / l^{2}\right)=1-\frac{\Gamma(2-p) 2^{3-2 p}}{\Gamma(p)} \sum_{r=1}^{\infty} \frac{e^{-\alpha_{r}^{2} x / l^{2} J_{1-p}\left(\alpha_{r}\right)}}{\alpha_{r}^{3-2 p} J_{p}\left(\alpha_{r}\right)} .
$$

For small values of $x / l^{2}$ the value of $\int_{0}^{x} E d x$ given by (30) reduces to the value (17) for the semi-infinite region, and

$$
\phi_{p}^{(1)}\left(x / l^{2}\right) \sim \frac{2^{2-2 p}\left(x / l^{2}\right)^{1-p}}{\Gamma(p)} .
$$

In Fig. 1 graphs of these quantities are shown for $p=1 / 9$, the value commonly found in wind tunnel experiments. Curve I shows the result (32) for the semi-infinite region, and Curve II the value of $\phi_{p}^{(1)}\left(x / l^{2}\right)$ given by (31) for values of $x / l^{2}$ for which the difference between (31) and (32) is important. For larger values of $x / l^{2}$ than those shown the exponentials in (31) are negligible.

In the case of heat transfer the quantity (30) gives the amount of heat taken up from the region 0 to $x$ of one of the planes.

5. The region $0<z<l . \chi^{(0)}(z)=0 . E \rightarrow E_{0}$, constant, as $z \rightarrow 0, x>0 . E=0, z=l$, $x>0$. This corresponds to the region $0<z<2 l$ with flow symmetrical about $z=l$, and with constant diffusion across the planes $z=0$ and $z=2 l$.

Here, proceeding as in Section 4, we find

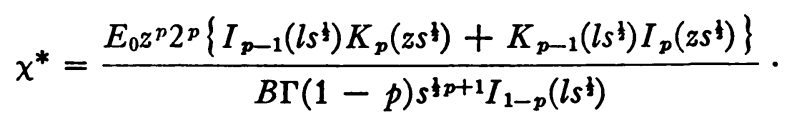

The most interesting quantity to evaluate in this case is the value of $\chi$ as $z \rightarrow+0$. This is found to be

$$
\frac{2 E_{0}(1-p) l^{2 p}}{B}\left[\psi_{p}\left(x / l^{2}\right)+\frac{x}{l^{2}}\right]
$$

' G. N. Watson, loc. cit., $\$ \$ 15.25,15.21$. For the method of calculating their values in practice see John R. Airey, Bessel functions of small fractional order and their application to problems of elastic stability, Phil. Mag. (6), 41, 200 (1921). 
where

$$
\psi_{p}\left(x / l^{2}\right)=\frac{1-p}{2 p(2-p)}-\frac{2^{2 p-1} \Gamma(p)}{\Gamma(2-p)} \sum_{r=1}^{\infty} \frac{e^{-x \alpha_{r}^{2} / l^{2} J_{p-1}\left(\alpha_{r}\right)}}{\alpha_{r}^{2 p+1} J_{-p}\left(\alpha_{r}\right)},
$$

and the $\alpha_{r}$ are the roots of

$$
J_{1-p}(\alpha)=0 .
$$

For small values of $x / l^{2},(34)$ tends to the result (19).

If $p=1 / 9$ it is found that the difference between (34) and (19) is less than $1 \%$ for values of $x / l^{2}$ up to 0.3 , while for greater values of $x / l^{2}$ the exponentials in (35) are

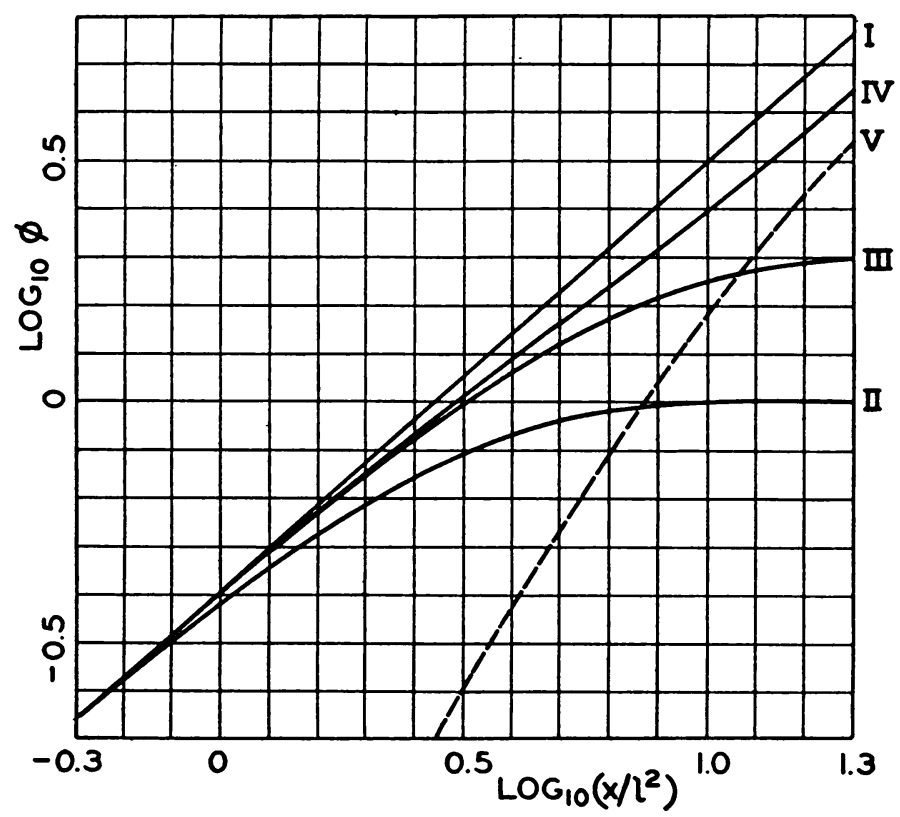

FIG. 1.

almost negligible. In the case of heat transfer, (34) gives the surface temperature of one of a pair of planes to which heat is supplied at a constant rate per unit time per unit area, and which are cooled by turbulent fluid flowing between them.

6. Two cases of symmetrical flow in the region $0<z<2 l$. First let us consider boundary conditions $\chi^{(0)}(z)=0$, and

$$
\begin{aligned}
& \chi=\chi_{0}, \quad \text { constant, when } z=0, x>0 \\
& E=0, \text { when } z=2 l, x>0 .
\end{aligned}
$$

Here the regions $0<z<l$ and $l<z<2 l$ must be treated separately. We write $\chi_{1}\left(z_{1}\right)$ and $E_{1}\left(z_{1}\right)$ for the values of $\chi$ and $E$ in $l<z<2 l$ as functions of $z_{1}=2 l-z$ in this region. The boundary conditions at the surface of separation $z=z_{1}=l$ are

$$
\chi=\chi_{1},
$$

$$
E=-E_{1}
$$


A solution of (9) which satisfies (37) is

$$
\chi^{*}=\frac{\chi_{0} 2^{1-p} s^{1 p-1}}{\Gamma(p)} z^{p} K_{p}\left(z s^{\natural}\right)+A z^{p} I_{p}\left(z s^{\natural}\right),
$$

and a solution of (9) with $z$ replaced by $z_{1}$ which satisfies (38) is

$$
\chi_{1}^{*}=C z_{1}^{p} I_{-p}\left(z_{1} s^{\ddagger}\right) \text {. }
$$

The unknowns $A$ and $C$ are found by substituting in the transforms of (39) and (40), which gives

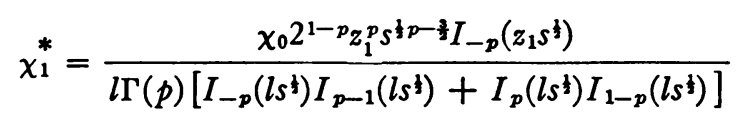

with a rather longer formula for $\chi^{*}$. As in Section $4, \chi$ and $\chi_{1}$ are evaluated by the use of the inversion theorem and the results are

$$
\begin{aligned}
& \chi_{1}=\chi_{0}-\frac{\chi_{0} 2^{1-p} z_{1}^{p} \Gamma(1-p)}{l^{p}} \sum_{r=1}^{\infty} \frac{e^{-x \alpha_{r}^{2} / l^{2}} \alpha_{r}^{p-1} J_{p-1}\left(\alpha_{r}\right) J_{p}\left(\alpha_{r}\right) J_{-p}\left(z \alpha_{r} / l\right)}{J_{p-1}^{2}\left(\alpha_{r}\right)+J_{p}^{2}\left(\alpha_{r}\right)}, \\
& \chi=\chi_{0}-\frac{\chi_{0} 2^{1-p} z^{p}}{l^{p} \Gamma(p)} \sum_{r=1}^{\infty} \frac{e^{-x \alpha_{r}^{2} / l^{2}} \alpha_{r}^{p-2} J_{p}\left(z \alpha_{r} / l\right)}{J_{p-1}^{2}\left(\alpha_{r}\right)+J_{p}^{2}\left(\alpha_{r}\right)}
\end{aligned}
$$

where the $\alpha_{r},(r=1,2, \cdots)$, are the positive $\operatorname{roots}^{10}$ of

$$
J_{-p}(\alpha) J_{p-1}(\alpha)-J_{p}(\alpha) J_{1-p}(\alpha)=0 .
$$

As in Section 4 the most interesting quantity is the value of $\int_{0}^{x} E d x$ as $z \rightarrow 0$. This is found to be

where

$$
\int_{0}^{x} E d x \rightarrow \frac{B \chi_{0} l^{2-2 p}}{2(1-p)} \phi_{p}^{(2)}\left(x / l^{2}\right)
$$

$$
\phi_{p}^{(2)}\left(x / l^{2}\right)=2-\frac{2^{3-2 p}(1-p)}{[\Gamma(p)]^{2}} \sum_{r=1}^{\infty} \frac{e^{-x \alpha_{r}^{2} / l^{2}}}{\alpha_{r}^{4-2 p}\left\{J_{p}^{2}\left(\alpha_{r}\right)+J_{p-1}^{2}\left(\alpha_{r}\right)\right\}} .
$$

For small values of $\left(x / l^{2}\right)$, (46) behaves like (32). Its value for $p=1 / 9$ and for values of $\left(x / l^{2}\right)$ for which the difference from (32) is important is shown in Curve III of Fig. 1 ; for larger values of $x / l^{2}$ the exponentials in (46) are negligible.

The result (45) gives the evaporation from the region 0 to $x$ of the plane $z=0$ if there is no flow over the plane $z=2 l$.

Finally we consider the case in which the boundary conditions are $\chi^{(0)}(z)=0$, and

$$
\chi=\chi_{0}, \text { constant, } z=0, x>0,
$$

and

$$
x=0 \text {, when } z=2 l, x>0 .
$$

Here, proceeding as before and writing $\chi_{1}$ for the value of $\chi$ in $l<z<2 l, E_{1}$ for the value of $E$ in this region, and $z_{1}=2 l-z$, we find

${ }^{10}$ In the case $k=1 / 2$ when (1) becomes the equation of linear flow of heat, (44) becomes $\cos 2 \alpha=0$ and similarly (51) and (52) become $\sin 2 \alpha=0$. 


$$
\begin{gathered}
\chi=\chi_{0}\left(1-\frac{z^{2 p}}{2 l^{2 p}}\right)-\frac{\chi_{0} 2^{1-p} z^{p}}{l p \Gamma(p)} \sum_{r=1}^{\infty}\left\{\frac{e^{-\alpha_{r}^{2} x / l^{2} J_{p}\left(z \alpha_{r} / l\right)}}{\alpha_{r}^{2-p} J_{p-1}^{2}\left(\alpha_{r}\right)}+\frac{e^{-\beta_{r}^{2} x / l^{2} J_{p}\left(z \beta_{r} / l\right)}}{\beta_{r}^{2-p} J_{p}^{2}\left(\beta_{r}\right)}\right\}, \\
\chi_{1}=\frac{1}{2} \chi_{0}\left(\frac{z_{1}}{l}\right)^{2 p}+\frac{\chi_{0} 2^{1-p} z_{1}^{p}}{l^{p} \Gamma(p)} \sum_{r=1}^{\infty}\left\{\frac{e^{-\alpha_{r}^{2} x / l^{2} J_{p}\left(z_{1} \alpha_{r} / l\right)}}{\alpha_{r}^{2-p} J_{p-1}^{2}\left(\alpha_{r}\right)}-\frac{e^{-\beta_{r}^{2} x / l^{2} J_{p}\left(z_{1} \beta_{r} / l\right)}}{\beta_{r}^{2-p} J_{p}^{2}\left(\beta_{r}\right)}\right\},
\end{gathered}
$$

where the $\alpha_{r}$ are the positive roots of

$$
J_{p}(\alpha)=0,
$$

and the $\beta_{r}$ are the positive roots of

$$
J_{p-1}(\beta)=0 .
$$

This problem is that of heat transfer between the plane $z=0$ at constant temperature $\chi_{0}$, and the plane $z=2 l$ at zero temperature, by turbulent fluid flowing between. The quantity of heat taken up from the region 0 to $x$ of the plane $z=0$ is determined by

where

$$
\lim _{s \rightarrow+0} \int_{0}^{x} E d x=\frac{B \chi_{0} l^{2-2 p}}{2(1-p)} \phi_{p}^{(3)}\left(x / l^{2}\right),
$$

$$
\begin{aligned}
\phi_{p}^{(3)}\left(x / l^{2}\right)= & \frac{2 p(1-p) x}{l^{2}}+\frac{1+p+2 p^{2}}{2(1+p)} \\
& -\frac{2^{3-2 p}(1-p)}{[\Gamma(p)]^{2}} \sum_{r=1}^{\infty}\left\{\frac{e^{-x \alpha_{r}^{2} / l^{2}}}{\alpha_{r}^{4-2 p} J_{p-1}^{2}\left(\alpha_{r}\right)}+\frac{e^{-x \beta_{r}^{2} / l^{2}}}{\beta_{r}^{4-2 p} J_{p}^{2}\left(\beta_{r}\right)}\right\}
\end{aligned}
$$

and the $\alpha_{r}$ and $\beta_{r}$ are defined by (51) and (52). For small values of $\left(x / l^{2}\right), \phi_{p}^{(3)}\left(x / l^{2}\right)$ behaves like (32). For larger values its behaviour for the case $p=1 / 9$ is shown in Fig. 1, Curve IV, and for still larger values the exponentials in (54) are negligible.

The quantity of heat taken up by the region 0 to $x$ of the plane $z=2 l$ is determined by

where

$$
-\lim _{x_{1} \rightarrow+0} \int_{0}^{x} E_{1} d x=\frac{B \chi_{0} l^{2-2 p}}{2(1-p)} \phi_{p}^{(4)}\left(x / l^{2}\right),
$$

$$
\begin{aligned}
& \phi_{p}^{(4)}\left(x / l^{2}\right)=\frac{2 p(1-p) x}{l^{2}}-\frac{(1+2 p)(1-p)}{2(1+p)} \\
& -\frac{2^{3-2 p}(1-p)}{[\Gamma(p)]^{2}} \sum_{r=1}^{\infty}\left\{\frac{e^{-x \alpha_{r}^{2} / l^{2}}}{\alpha_{r}^{4-2 p} J_{p-1}^{2}\left(\alpha_{r}\right)}-\frac{e^{-x \beta_{r}^{2} / l^{2}}}{\beta_{r}^{4}{ }^{2 n} J_{p}^{2}\left(\beta_{r}\right)}\right\} \text {. }
\end{aligned}
$$

A portion of the curve of $\phi_{p}^{(4)}\left(x / l^{2}\right)$ for $p=1 / 9$ is shown in Fig. 1, Curve V; for larger values of $x / l^{2}$ the exponentials in (56) are negligible, and for smaller values than those shown in the figure $\phi_{p}^{(4)}$ continues to decrease rapidly. 